\title{
Analyse de l'écoulement au voisinage d'un débitmètre à vortex pour améliorer la précision de comptage en milieu industriel
}

\author{
A. Strzelecki*, P. Gajan*, M. Bosch**, D. Donat*** \\ * Centre d'Etudes et de recherches de Toulouse \\ Office National d'Etudes et de Recherches Aérospatiales \\ ** Gaz du Sud-Ouest \\ *** Gaz de France
}

Etant donné les quantités de gaz importantes échangées chaque jour entre partenaires industriels, les sociétés gazières responsables du transport veillent à améliorer la précision du comptage. Cette communication présente la méthodologie originale suivie par des chercheurs du Centre d'Etudes et de Recherches de Toulouse (CERT/ONERA) depuis 15 ans pour le compte de Gaz de France et de Gaz du Sud-Ouest.

Les débitmètres utilisés sur des installations industrielles sont étalonnés dans des conditions d'écoulement de référence correspondant à un écoulement turbulent pleinement développé en conduite. Pour obtenir de telles conditions, il est nécessaire de placer à l'amont du débitmètre des longueurs droites de tube rectiligne importantes pouvant atteindre 100 diamètres de conduite.

Sur un site industriel, ces distances ne sont pas toujours disponibles, et une des principales causes d'erreur provient des modifications d'écoulement engendrées par les différents éléments placés à l'amont du débitmètre (coude, combinaison de coudes, vanne, pompe, compresseur, etc...).

Une approche classique consiste à étudier les erreurs de comptage associées à différents organes perturbateurs placés à l'amont des débitmètres. L'inconvénient majeur de cette approche est de ne pas améliorer la compréhension des phénomènes et la transposition des résultats à des configurations industrielles voisines est délicate.
Afin de remédier à cette situation le CERT/ONERA propose une démarche différente. Dans les travaux réalisés au CERT, les écoulements perturbés présents sur les sites industriels sont décomposés en perturbations élémentaires (dissymétrie, profil symétrique, rotation, pulsation, etc...). Leurs influences sur les débitmètres (diaphragmes, turbines, vortex, ultrasons, etc...) sont ensuite étudiées séparément à partir de perturbations calibrées. Nos recherches utilisent une approche expérimentale (visualisation, traitement d'images, mesures classiques (LDA)) et une approche numérique (Random Vortex Method, Large Eddy Simulation, etc...).

Dans ce papier, nous proposons d'illustrer notre démarche en caractérisant l'influence de certaines perturbations calibrées (dissymétrie du profil de vitesse, pulsation et rotation d'écoulement) sur un débitmètre vortex. Nous présenterons des visualisations d'écoulement illustrant les phénomènes, les résultats expérimentaux seront comparés à ceux issus de simulations d'écoulement.

Le principe de fonctionnement d'un débitmètre à vortex consiste à placer dans la conduite, normalement à l'écoulement, un obstacle de hauteur « $d »$. En présence d'un écoulement, il se produit en aval de l'obstacle une double rangée de tourbillons se détachant de façon alternée à une fréquence " $F_{\mathrm{rs}}$ " proportionnelle à la vitesse débitante $U$ du fluide. La relation entre la fréquence de détachement, la

Flow analysis near a vortex-ring flowmeter in order to improve counting precision in the industry

This paper presents the inventive methodology of CERTIONERA searchers working for Gaz de France and Gaz du Sud-Ouest, in order to improve counting precision in the industry. The authors characterize the effects of some gauged disturbances (speed profile dissymetry, flow pulsation and rotation) on a vortex flowmeter. Experimental results are compared to flow simulation results. 
vitesse débitante et la hauteur du barreau s'effectue au travers d'un nombre adimensionnel, le nombre de Strouhal $\left(\mathrm{St}=\frac{F_{t s} \cdot d}{U}\right)$, qui reste constant pour une large gamme du nombre de Reynolds $\left(\operatorname{Re}=\frac{U \cdot d}{v}\right)$. Ainsi à partir d'une mesure de fréquence, il est possible de déterminer le débit de fluide s'écoulant dans la canalisation. Comme pour tout débitmètre, les caractéristiques de l'écoulement vont influer sur cette relation : ceci fait l'objet de cette communication.

\section{Moyens}

\subsection{Bancs d'expérimentation}

\section{I.I.I. Banc hydraulique A veine de section rectangulaire)}

Le tunnel hydrodynamique présenté sur la figure I a été utilisé pour caractériser l'écoulement dans la maquette bidimensionnelle du débitmètre vortex pour un écoulement amont non perturbé, dissymétrique ou pulsé. La description détaillée de ce tunnel est présentée dans Strzelecki [1]. Il comporte une veine d'essai en plexiglas d'un mètre de long et de section rectangulaire $(150 \mathrm{~mm} \times 180 \mathrm{~mm})$. La hauteur de la veine d'essai peut prendre respectivement trois valeurs : $D=180,120$ et $90 \mathrm{~mm}$. La hauteur $d$ de l'obstacle mesure $30 \mathrm{~mm}$. Il est ainsi possible de modifier le blocage de l'obstacle sans modifier l'allongement $(d / L)$.

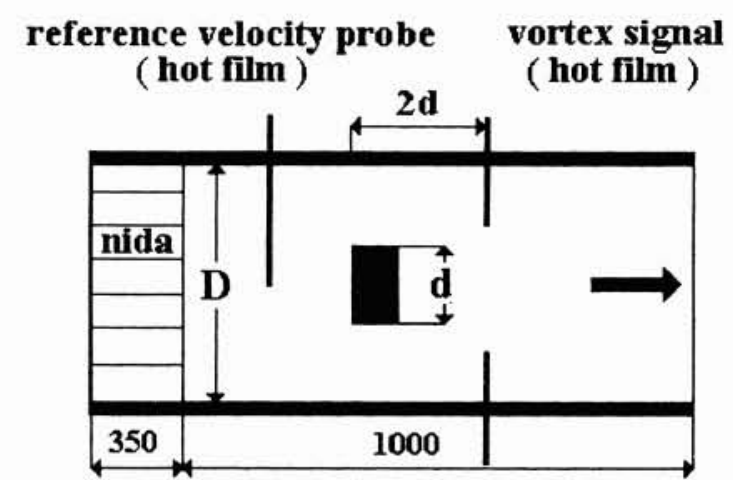

$d=30 \mathrm{~mm} ; D=90,120$ or $180 \mathrm{~mm}$

1. Veine d'essai 2 D; hydraulique.

Une dérivation de l'écoulement, située entre la pompe et la veine d'essai, traverse un générateur de pulsation : celui-ci engendre une variation périodique du débit, provoquée par une obturation alternative de la section de l'écoulement. Le débit dans la veine d'essai est contrôlé par un débitmètre à ultrasons. Le débit maximum est de $10 \mathrm{l} / \mathrm{s}$ et la fréquence de pulsation peut varier de 0,2 à $2 \mathrm{~Hz}$. L'amplitude des pulsations est réglable. Les fréquences de pulsation de l'écoulement et du signal vortex sont mesurées à l'aide de trois capteurs (optique, compteur à ultrasons et sondes à film chaud). Les mesures de vitesse ont été effectuées par vélocimétrie laser ou anémométrie à film chaud.

\section{I.1.2. Banc hydraulique B (veine de section circulaire)}

Les essais destinés à caractériser l'influence d'une rotation d'écoulement sur un débitmètre à vortex ont été effectués sur ce banc hydrodynamique. Une description détaillée de cette boucle est faite dans Gajan et al.[2]. La maquette du débitmètre à vortex dont le diamètre intérieur est égal à $100 \mathrm{~mm}$ est placée à 47 diamètres d'un réservoir fermé utilisé pour éliminer les pulsations générées par la pompe. Le débit total est mesuré à l'aide d'un débitmètre électromagnétique $\left(Q_{\max }=140 \mathrm{~m}^{3} / \mathrm{h}\right)$ placé à l'amont du réservoir de tranquillisation. Pour ces essais le générateur de swirl est placé entre ce réservoir et la maquette de mesure. Pour ce type de générateur, le débi total $Q_{\text {total }}$ est divisé en deux débits : $Q_{a}$ le débit axial et $\mathcal{C}_{c}$. le débit tangentiel. Ce dernier est injecté tangentiellement tu débit axial mettant ainsi le fluide en rotation. La répartition relative des deux débits est contrôlée à l'aide de deux vannes. Un deuxième débitmètre électromagnétique permet de mesurer le débit axial $Q_{a}$. L'intensité de la rotation induite est alors déterminée à partir d'un nombre sans dimension $\Omega$ défini par :

$$
\Omega=1-\frac{Q_{\text {axial }}}{Q_{\text {total }}} .
$$

L'obstacle du débitmètre vortex est placé à 18 diamètres à l'aval du générateur de swirl. Il est de forme trapézoïdale (hauteur frontale $d=23 \mathrm{~mm}$, blocage $B=0,293$ ).

Pour ces essais, la fréquence de détachement tourbillonnaire à l'aval de l'obstacle est déterminée à partir d'une mesure de vitesse par un anémomètre film chaud. La sonde est placée à $1,8 d$ de la face aval de l'obstacle et à $0,83 d$ de laxe de la conduite.

\subsubsection{Banc aérodynamique}

Le banc aérodynamique a permis de caractériser l'influence de trois types de perturbation amont sur le débitmètre industriel (dissymétrie, pulsation, détendeur). Une description détaillée de ce moyen d'essai est faite dans Strzelecki [3]. Il est constitué d'un réservoir pressurisé amont haute pression ( 160 bars), d'un col sonique à section variable (débitmètre de référence), d'une veine d'essai circulaire (diamètre $=100 \mathrm{~mm}$ ). Une longueur droite de 47 diamètres à l'amont de la veine d'essai assure dans cette section un profil de vitesse pleinement développé. Les caractéristiques géométriques du débitmètre vortex utilisé sont identiques à celles spécifiées dans 2.1.2 (diamètre intérieur $D=100 \mathrm{~mm}$, $d=23 \mathrm{~mm}, B=0,293$ ).

\subsection{Visualisation d'écoulement}

Pour visualiser les écoulements hydrauliques, nous utilisons deux types de traceurs : des traceurs gazeux (bulles d'air ou d'hydrogène) et des traceurs liquides (colorants). Dans chaque cas, l'écoulement est éclairé par une tranche lumineuse et observé latéralement au moyen d'une caméra vidéo.

De petites bulles d'air sont générées en ajoutant du teepol dans l'eau. Les micro-bulles apportent des informations qualitatives sur le détachement tourbillonnaire. Il est possible de superposer en temps réel aux images vidéo deux signaux reliés au détachement tourbillonnaire : le signal de vitesse vortex (film chaud) et le signal de vitesse de référence à l'amont de l'obstacle.

Les bulles d'hydrogène sont produites de façon contrôlée à partir d'un fil de tungstène $(100 \mu \mathrm{m})$ servant de cathode : 
leur génération peut être continue ou synchronisée avec la pulsation d'écoulement. Un exemple de visualisation d'écoulement obtenu avec cette technique est présentée sur la figure 3.

Différents types de colorants (rhodamine, rhodorsil ou fluoresceine), injectés au moyen de petits trous situés sur l'obstacle permettent de visualiser les contours tourbillonnaires et d'étudier leur mouvement (fig. 2).

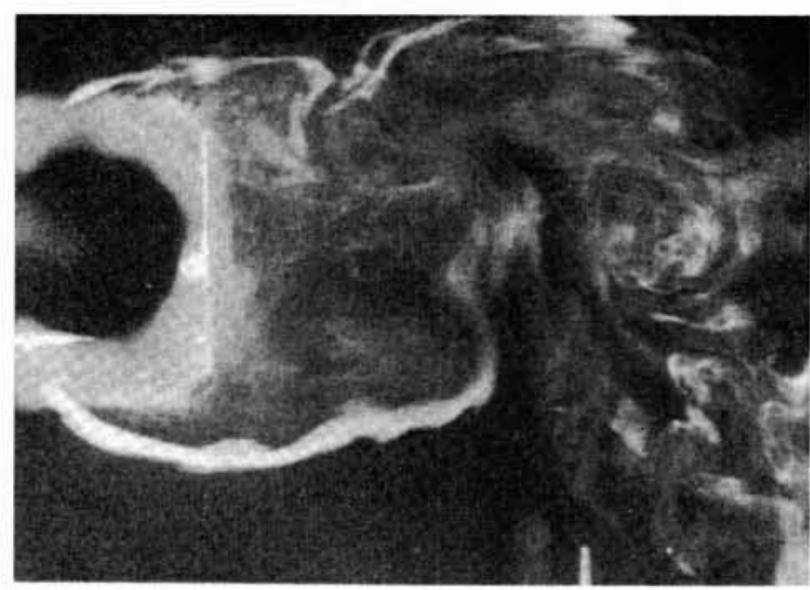

2. Visualisation par colorant.

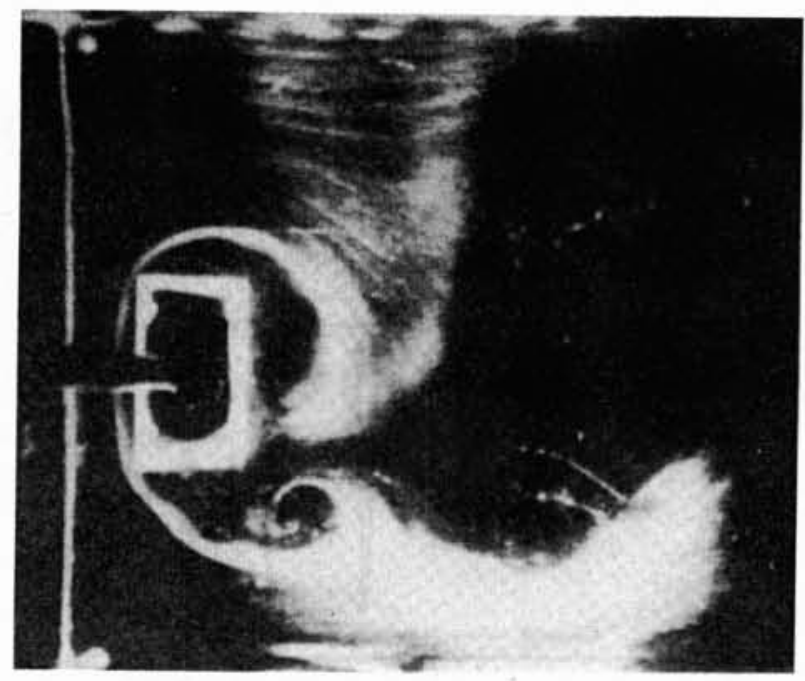

3. Visualisation par bulle d'hydrogène en écoulement pulsé.

\subsection{Traitement du signal}

Le signal de vitesse est traité à l'aide d'un analyseur spectral (B \& K-2032) afin d'obtenir la fréquence de détachement $f_{v s}$ et de déterminer la qualité du signal. La qualité $Q$ du signal est égale au rapport entre l'énergie dans une bande $\Delta f(=1,4 \mathrm{~Hz})$ centrée autour du maximum de la raie et l'énergie totale. L'énergie dans une bande $\Delta f$ est diminuée par le bruit en supposant que ce bruit est identique sur tout le spectre :

$$
Q=\frac{\left[R M S^{2}-R M S_{\text {bruit }}^{2}\right]^{1 / 2} \Delta f}{R M S_{\text {Total }}} .
$$

Cette quantité peut être reliée au rapport signal sur bruit $(S / N)$ par la relation suivante :

$$
S / N=1+\frac{Q}{\left(1-Q^{2}\right)^{1 / 2}} .
$$

Pour compléter cette analyse donnant la fréquence de détachement et son énergie, une analyse temps-fréquence par l'utilisation de la transformée en ondelettes continues a été réalisée. Ces résultats permettent de montrer le caractère intermittent du phénomène. Nous ne détaillerons pas ici cette méthode de traitement du signal reposant sur une convolution du signal avec une fonction oscillante appelée ondelette localisée en temps et en fréquence. Pour plus de renseignements à son sujet il est possible de se référer aux travaux de Martinez [4].

\subsection{Simulations numériques}

\subsubsection{La méthode RVM (Random Vortex Method)}

La méthode RVM, introduite par Chorin, est applicable à la simulation des écoulements turbulents. Cette méthode, particulièrement adaptée à la modélisation des grosses structures turbulentes, est développée au CERT/ONERA depuis plusieurs années. Des détails concernant cette méthode de simulation sont donnés dans Blot et al. [5]. Elle a permis en particulier de caractériser l'influence de plusieurs paramètres sur le détachement tourbillonnaire (nombre de Reynolds, blocage, profil de vitesse amont).

\subsubsection{La méthode LES/FCT (Large Eddy Simulation/Flux Corrected Transport)}

La technique de simulation utilisée repose sur une méthode aux volumes finis. Le schéma numérique de discrétisation des équations est basé sur une technique de Transport par Flux corrigés (Flux Corrected Transport en anglais) développée dans les années 70 par Boris et Book [6] au NRL de Washington. Depuis, cette approche a largement été utilisée pour la simulation de jet plan et axisymétrique compressible subsonique instationnaire turbulent, pour des couches de mélanges supersoniques ou pour des problèmes d'écoulements diphasiques réactifs.

Pour plus de détails concernant cette méthode de calcul nous renvoyons le lecteur vers les travaux de Berger et Estivalezes [7], Rompteaux [8] et Berger et al. [9].

L'obstacle est de forme rectangulaire avec un rapport d'allongement (épaisseur/hauteur) de 0,5 . Il est placé à trois hauteurs de la section initiale du calcul. Les signaux de vitesse ayant servi à calculer la fréquence de détachement sont pris en un point du domaine situé transversalement à une hauteur d'obstacle de l'axe et à deux hauteurs de la face amont du barreau. Pour ce calcul, la vitesse débitante est de $35 \mathrm{~m} / \mathrm{s}$, la masse volumique est de $1,189 \mathrm{~kg} / \mathrm{m}^{3}$ et la pression moyenne de 1 bar. En écoulement pulsé la vitesse d'entrée suit une loi sinusoïdale de la forme :

$$
U(t)=U_{0}(1+\tau \sin (\omega t)) .
$$




\section{Résultats}

Avec les moyens présentés dans le paragraphe précédent, le comportement du débitmètre vortex est caractérisé en présence de plusieurs perturbations d'écoulement par comparaison à un écoulement stationnaire non perturbé. Les écoulements traités seront

- Ecoulement amont stationnaire non perturbé

- Ecoulement amont stationnaire avec des profils de vitesse dissymétriques.

- Ecoulement amont en giration.

- Ecoulement pulsé.

\subsection{Régime d'écoulement stationnaire non perturbé}

De nombreux résultats ont été obtenus avec les deux types de débitmètre vortex (2D ou 3D). Ils ont permis de caractériser le phénomène de détachement tourbillonnaire en terme de nombre de Strouhal, de stabilité et de rapport signal sur bruit en fonction de différents paramètres comme le nombre de Reynolds et le blocage. Les résultats obtenus servent ensuite de référence afin d'étudier l'influence des perturbations.

Les visualisations présentées sur les figures 2 et 3 révèlent la structure de l'écoulement au voisinage de l'obstacle prismatique. Pour différentes valeurs du blocage et du nombre de Reynolds, le signal de vitesse vortex est analysé et nous obtenons les principales conclusions suivantes :

- Chaque spectre comporte deux pics dont la largeur de bande est représentative de l'instabilité du signal et dépend du nombre de Reynolds. Le premier correspond à la fréquence de détachement tourbillonnaire $F_{t s}$ alors que le second $\left(2 F_{r s}\right)$ est associé au détachement tourbillonnaire alterné.

- Le phénomène de détachement tourbillonnaire peut être détruit pour quelques périodes et conduire dans ces conditions à une disparition du signal.

La figure 6 montre que le nombre de Strouhal est une fonction croissante du blocage. Par exemple, pour une gamme de nombre de Reynolds compris entre $5.10^{3}$ et I.7.10 $10^{4}$. les valeurs du nombre de Strouhal $\mathrm{St}=0.20$ et $\mathrm{St}=0.17$ mesurées pour des blocages respectifs de $B=0,25$ et $B=0,17$ sont en bon accord avec celles de la littérature ou obtenues par la méthode RVM (figs. 4 et 6).

Un résultat similaire est obtenu à partir de la méthode de simulation LES/FCT (fig. 5) pour un blocage $B=0,33$. Le nombre de Strouhal obtenu est alors égal à 0,223 .

Pour un blocage $B=0,29$, correspondant au débitmètre industriel testé (géométrie 3D), le nombre de Strouhal expérimental (expériences hydrauliques et aérodynamiques) est en accord avec les résultats $2 \mathrm{D}$. On peut ainsi noter que, pour une même géométrie de barreau, le paramètre de blocage permet de passer d'une configuration 2D à une configuration 3D.

\subsection{Régime d'écoulement stationnaire dissymétrique}

La dissymétrie de l'écoulement amont est obtenue à l'aide d'un générateur à faisceau de tubes. Cette dissymétrie est placée soit perpendiculairement au barreau, soit parallèlement.

Les résultats de ces mesures ont mis en évidence une redistribution du profil de vitesse qui tend à devenir plus uniforme. Cet effet est d'autant plus marqué que le blocage augmente. Par conséquent, l'accroissement du confinement

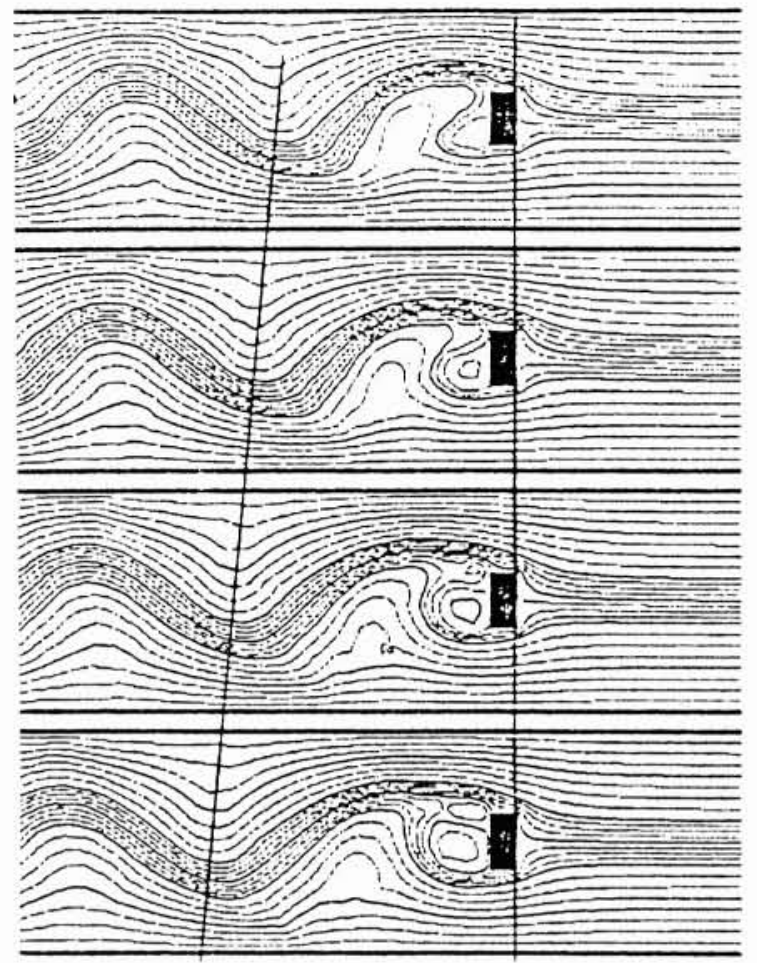

4. Méthode RVM ; lignes de courant, blocage $=0,25$.

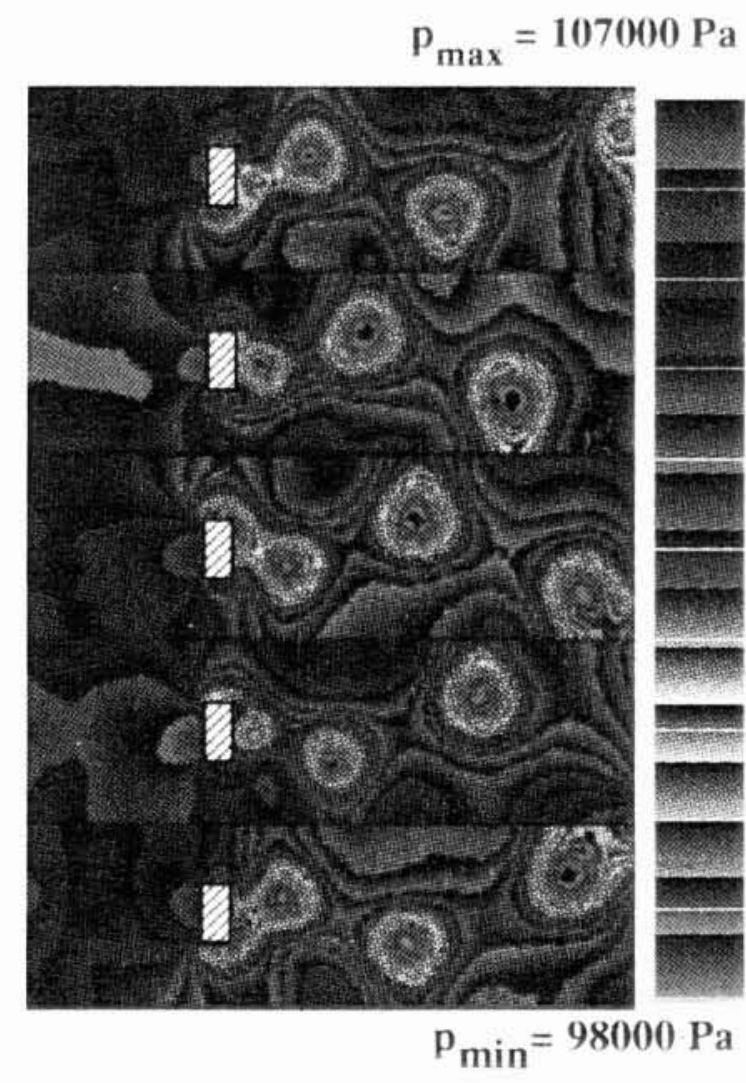

5. Méthode LES, champ de pression instantané en écoulement amont stationnaire $;$ blocage $=\mathbf{0 , 3 3}$. 
- cert ( 20 ; R.V.M. ; exp. profile)

* cert ( 20 ; R.V.M. ; llat profile)

- cert (2D; hydraulic)

- cert ( 30 ; aerodynamic)

- Bouras

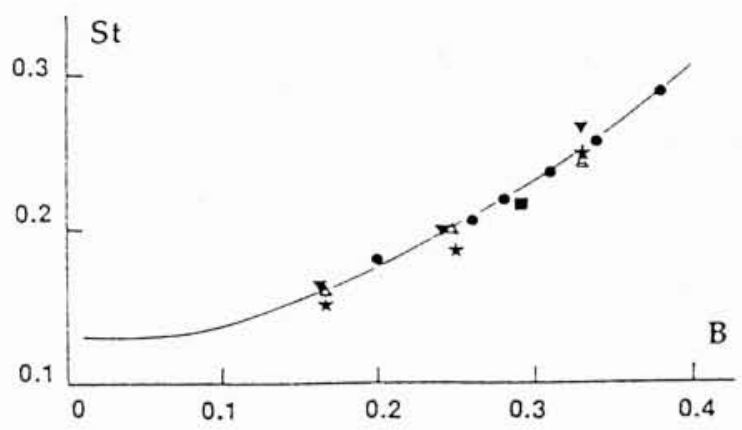

6. Nombre de Strouhal en fonction du blocage.

tend à minimiser l'influence de la forme du profil de vitesse amont (fig. 7).

Des dissymétries importantes de vitesse dans un plan perpendiculaire à l'axe de l'obstacle conduisent à des écarts relativement faibles (de l'ordre de $2 \%$ ) sur le nombre de Strouhal dû à l'effet de "filtre " exercé par le blocage (fig. 7).

Lorsque la dissymétrie est parallèle à l'envergure du barreau, le phénomène de détachement devient beaucoup plus instable et deux fréquences voisines apparaissent sur les spectres (fig. 8). Ceci rend difficile un traitement du signal adapté.

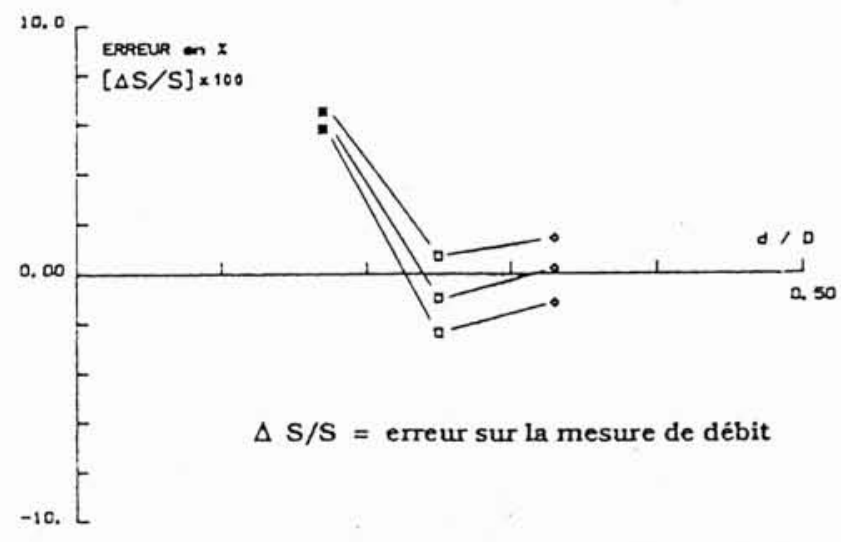

7. Erreur relative en fonction du blocage pour un profil de vitesse amont dissymétrique dans un plan perpendiculaire à l'axe de l'obstacle.

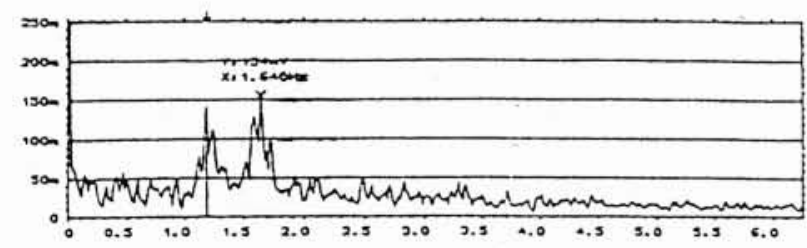

8. Influence d'une dissymétrie de vitesse dans un plan parallèle à l'axe de l'obstacle ; analyse spectrale des fluctuations.

\subsection{Régime d'écoulement stationnaire avec rotation}

\subsubsection{Analyse des signaux en fonction de l'intensité de la rotation}

Les essais ont été menés pour une valeur du nombre de Reynolds $\operatorname{Re}_{D}$ égal à 145000 . Les spectres obtenus pour quatre intensités de rotation $(\Omega=0 ; 0,32 ; 0,41 ; 0,58)$ montrent une dégradation de la qualité du signal au fur et à mesure que l'intensité de la rotation augmente.

Sur la figure 9 l'évolution de la fréquence de détachement tourbillonnaire $F_{t y}$ en fonction de l'intensité de la rotation est représentée. Sur cette figure, la fréquence de détachement est présentée sous forme du nombre de Strouhal St. Pour des valeurs de $\Omega$ inférieures à 0,5 , une raie correspondant au phénomène de détachement tourbillonnaire émerge du bruit.

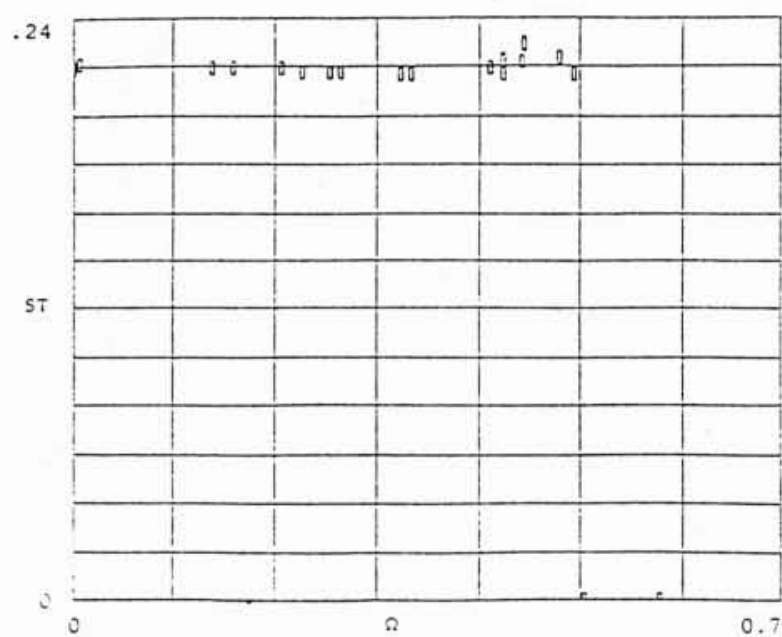

9. Nombre de Strouhal en fonction du niveau de rotation $\left(\operatorname{Re}=1,45 \times 10^{5}\right)$.

La valeur du nombre de Strouhal associé reste constante avec des écarts ne dépassant pas 0,0036 pour une valeur moyenne de 0,2202 . Cette dernière valeur est en bon accord avec les résultats précédents obtenus dans l'air avec la même géométrie d'obstacle [1].

Sur la figure 10 est présentée l'évolution de la qualité $Q$ du signal en fonction de $\Omega$. Ces valeurs sont normalisées par la qualité du signal obtenue sans rotation. Pour cette valeur du nombre de Reynolds, la qualité du signal reste identique à celle obtenue sans rotation pour des valeurs de $\Omega$ allant jusqu'à 0,2 . Entre 0,2 et 0,3 , la qualité augmente légèrement. Au-delà, $Q$ diminue fortement jusqu'à disparition du signal.

Cette analyse est complétée à l'aide d'une transformation en ondelettes qui permet de décomposer le signal temporel en deux dimensions temps-fréquence. L'utilisation de cette technique de traitement montre nettement l'apparition de phénomènes intermittents de plus en plus marqués au fur et à mesure que l'intensité de la rotation amont augmente. Ce phénomène s'accompagne d'un élargissement de la bande fréquentielle contenant l'énergie du signal.

A partir du résultat brut de cette transformée, un posttraitement détaillé dans [4] a permis de définir, pour chaque signal étudié, un coefficient d'intermittence et un taux de fluctuation de la fréquence de détachement tourbillonnaire. 


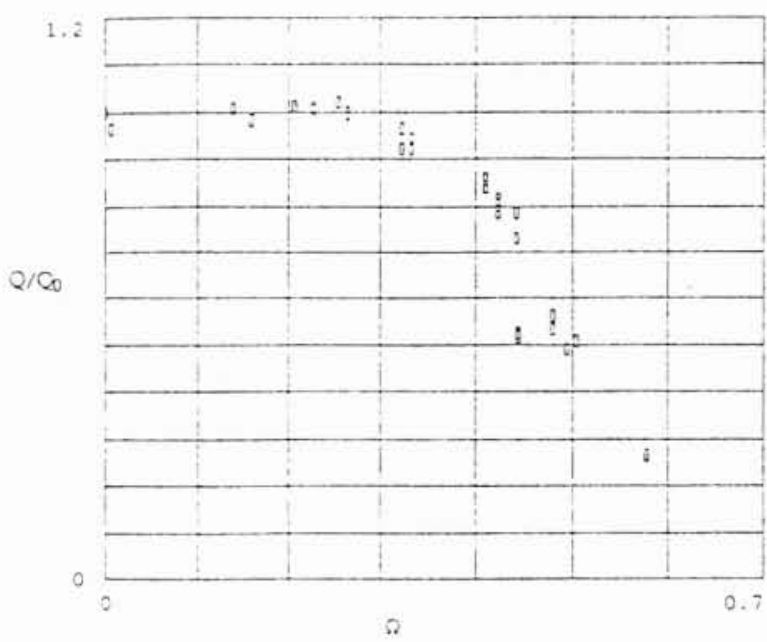

10. Qualité du signal en fonction du niveau de rotation $\left(\operatorname{Re}=1,45 \cdot 10^{5}\right)$.

A partir du résultat de ces différentes méthodes d'analyse, il est possible de donner les conclusions suivantes :

- Pour le nombre de Reynolds considéré, le phénomène de détachement tourbillonnaire existe pour des valeurs de $\Omega$ pouvant atteindre 0,5 .

- Tant que le phénomène de détachement peut être détecté, le nombre de Strouhal reste sensiblement constant et égal à celui obtenu sans rotation.

- Tant que le phénomène de détachement peut être détecté, le nombre de Strouhal reste sensiblement constant et égal à celui obtenu sans rotation.

- Pour $\Omega$ inférieur à 0,3 , la qualité du signal reste constante. L'analyse par ondelette révèle même une amélioration du signal pour $\Omega=0.25$ (diminution de l'intermittence. diminution de la fluctuation de la fréquence).

- Pour des valeurs de $\Omega$ supérieures, la qualité du signal s'appauvrit, se traduisant par une augmentation de l'intermittence et de la fluctuation de la fréquence de détachement.

\subsubsection{Influence du nombre de Reynolds}

Des essais similaires à ceux présentés dans la partie précédente ont été réalisés pour d'autres valeurs du nombre de Reynolds comprises entre 25000 et $150000[10]$. Ces résultats montrent des comportements similaires à ceux décrits précédemment.

Néanmoins, il a été observé que la valeur du nombre de Reynolds influait sur la valeur de $\Omega$ critique pour laquelle le signal vortex commençait à se détériorer. On obtient ainsi une détérioration plus rapide du phénomène de détachement tourbillonnaire lorsque le nombre de Reynolds augmente.

\subsection{Régime d'écoulement pulsé}

De nombreux tests ont été réalisés aussi bien sur une géométrie 2D dans l'eau que 3D dans l'air. Les paramètres d'analyse sont

- Le blocage $B$.

- Le nombre de Reynolds Re.

- La fréquence de pulsation $F_{p}$.

- L'amplitude de la pulsation $T_{p}$.

Pour chaque test, on fixe le nombre de Reynolds et l'amplitude de la pulsation. Seule la fréquence de pulsation est modifiée. Les résultats présentés sur la figure $/ /$ font apparaitre les modes suivants :

- Pour $F_{p} / F_{r s}<1$, deux raies sont présentes sur les spectres pour $F_{p}$ et $F_{r p}$ où $F_{r p}$ est la fréquence de détachement en régime pulsé. Cette fréquence est proche de la fréquence de détachement en régime stationnaire $F_{t s}$, ce qui n'entraîne pas d'erreur significative sur la mesure de débit. - Pour $F_{p} / F_{\mathrm{rs}} \sim 1$, une raie est présente sur les spectres pour $F_{n}=F_{r p}$. Pour ce phénomène qui n'apparaît que pour des taux de pulsation importants $(>15 \%)$ deux modes instables peuvent être présents par intermittence. Le mode 1 qui est un mode de détachement alterné à la fréquence $F_{r p}=F_{p}$. L'erreur de comptage est alors $\left(\varepsilon=\frac{\Delta F}{F}\right)$. La plage d'accrochage est une fonction croissante du taux de pulsation. Ce mode est plus présent avec de faibles blocages. Le mode 2 est un mode de détachement symétrique. II entraîne une disparition du signal si la détection des tourbillons est basée sur une différence de phase entre les deux allées.

- Pour $F_{p} / F_{t s}-2$, I'accrochage qui correspond au mode 3 est toujours présent. La plage d'accrochage est une fonction croissante du taux de pulsation. Ce mode qui est très stable correspond à un détachement non symétrique de tourbillon à une fréquence $F_{r p}=2 F_{p}$. L'erreur de comptage peut être très importante. Dans ce cas également, une augmentation du blocage réduit l'effet.

Des résultats similaires ont été obtenus par d'autres auteurs.

Nous avons également étudié ces phénomènes d'accrochage à partir de simulations. Des résultats comparables aux expériences ont ainsi été obtenus à l'aide de la méthode LES/FCT pour un mode d'accrochage de type 3 .

a)

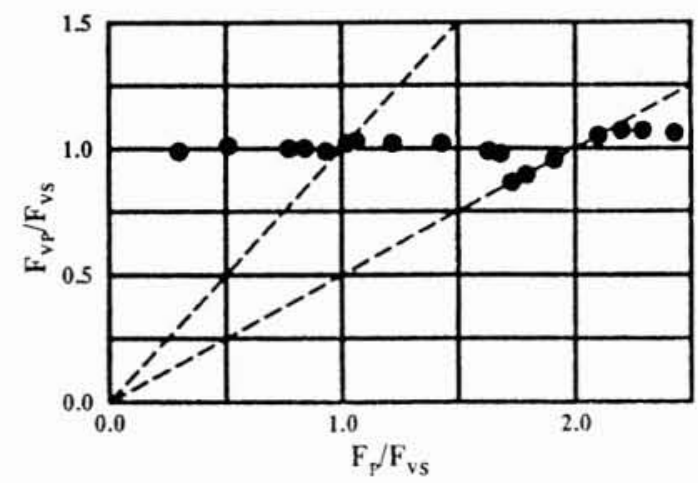

b)

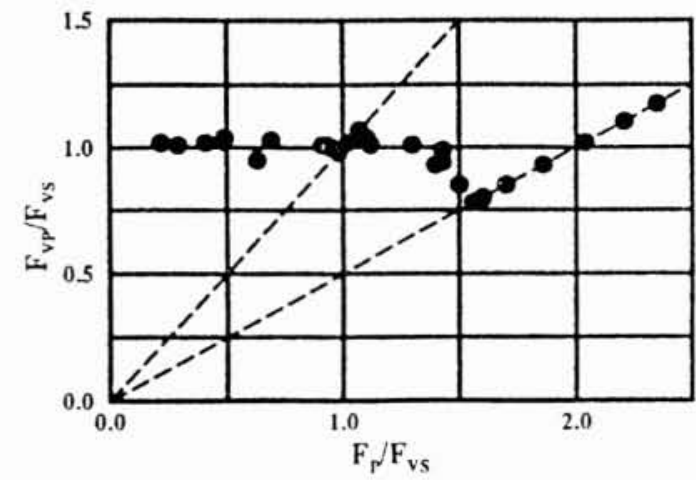

11. Fréquence de détachement tourbillonnaire en écoulement pulsé en fonction de la fréquence de pulsation ; a) $T_{p}=0,10$; b) $T_{p}=0,20$. 


\section{A. STRZELECKI, P. GAJAN, M. BOSCH, D. DONAT}

\section{Conclusion}

Les différentes approches suivies au CERT/DERMES sur les problèmes de comptage de fluide permettent de mieux comprendre les écoulements dans les conduites industrielles et leurs répercussions sur la mesure de débit. Les perturbations réelles présentes à l'aval de coudes ou de détendeurs sont décomposées en perturbations élémentaires stationnaires ou instationnaires dont les influences sont étudiées séparément. Dans le cas présenté ici concernant la débitmétrie par compteur à vortex, on a pu chiffrer ainsi les erreurs introduites et définir des niveaux admissibles ne dégradant pas la mesure. Toutes ces informations quantitatives sont ensuite utilisées pour analyser les résultats obtenus en configuration réelle, comme par exemple à l'aval d'un détendeur, et pour déterminer les conditions d'installation (longueur droite nécessaire, position du barreau par rapport au plan d'un coude). Ces résultats pratiques permettent d'améliorer la précision de comptage sur toutes les installations industrielles.

Cette approche d'ensemble est également appliquée à d'autres géométries de débitmètres.

\section{Bibliographie}

[1] Strzelecki A., Gajan P., Hebrard P. - Experimental analysis of flow phenomena in a vortex flowmeter in steady and pulsatile flow conditions. In Flomeko'89, Dusseldorf (1989).
[2] Gajan P., Hébrard P., Millan P., Giovannini A., Al. Isbert A., Strzelecki A., Trichet P. - Etude fondamentale sur la débitmétrie des gaz par diaphragme, rapport CERT/DERMES/14/2314 (1991).

[3] Strzelecki A. - Etude du détachement tourbillonnaire en écoulement perturbé : application à la débitmétrie vortex. Doctorat de l'Université Paul Sabatier, Toulouse (1989).

[4] Martinez J. - Utilisation de la méthode d'analyse par ondelettes pour la caractérisation des structures cohérentes en écoulement turbulent, Rapport DRET 901 563/J000/DRET/DS/SR (1992).

[5] Blot F., Giovannini A., Hebrard P., Strzelecki A. - Flow analysis in a vortex flowmeter : an experimental and numerical approach, In Turbulent Shear Flow, Stanford (1989).

[6] Boris J.P., BoOK D.L., SHATA, - A fluid transport algorithm that works, Journal of computational physics, 11. pp. 38-69 (1973).

[7] Berger C., Estivalezes J.L. - Aérodynamique interne des propulseurs à poudre, Rapport final CERT 242400/MES (1991).

[8] Rompteaux A. - Développement d'un code de calcul instationnaire compressible en volumes finis à faible diffusivité numérique, Doctorat de l'ENSAE, Toulouse (1992).

[9] Berger C., Estivalezes J.L., Gajan P., Perrin C., ROMPTEAUX A., STRZFlecki A. - Vortex shedding at pulsating flow conditions: an experimental and numerical approachs, Turbulent Shear Flows, Kyoto (1993).

[10] Laneville A., Martinez J., Strzelecki A., Gajan P. - Vortex flowmeter exposed to swirling flows: further results, soumis à Flucome'94, Toulouse (1994). 